\title{
BMJ Open Discrepancies between self-reported hearing difficulty and hearing loss diagnosed by audiometry: prevalence and associated factors in a national survey
}

Ji Eun Choi, ${ }^{1}$ II Joon Moon, ${ }^{2}$ Sun-Young Baek, ${ }^{3}$ Seon Woo Kim, ${ }^{3}$ Yang-Sun Cho ${ }^{2}$

To cite: Choi JE, Moon IJ, Baek S-Y, et al. Discrepancies between self-reported hearing difficulty and hearing loss diagnosed by audiometry: prevalence and associated factors in a national survey. BMJ Open 2019;9:e022440. doi:10.1136/ bmjopen-2018-022440

- Prepublication history for this paper is available online. To view these files, please visit the journal online (http://dx.doi org/10.1136/bmjopen-2018022440).

JEC and IJM contributed equally.

Received 21 February 2018 Revised 27 February 2019 Accepted 1 March 2019

Check for updates

(C) Author(s) (or their employer(s)) 2019. Re-use permitted under CC BY-NC. No commercial re-use. See rights and permissions. Published by BMJ.

For numbered affiliations see end of article.

Correspondence to Professor Yang-Sun Cho; yscho@skku.edu

\section{ABSTRACT}

Objective To evaluate discrepancies prevalent between self-reported hearing difficulty (SHD) and audiometrically measured hearing loss (AHL) and factors associated with such discrepancies.

Design Nationwide cross-sectional survey. Setting Data from 2010 to 2012 Korea National Health and Nutrition Examination Survey conducted by the Korea Centers for Disease Control and Prevention.

Participants We included 14345 participants aged $\geq 19$ years who had normal tympanic membranes (mean age of 49 years).

Measures Self-reported hearing was assessed by asking participants whether they had difficulty in hearing. AHL was defined as $>25 \mathrm{~dB}$ of mean hearing thresholds measured at $0.5,1,2$ and $4 \mathrm{kHz}$ in better ear. Underestimated hearing impairment (HI) was defined as having AHL without SHD. Likewise, overestimated HI was defined as having SHD without AHL. Prevalence of underestimated and overestimated HIs was determined. Univariable and multivariable analyses were performed to examine factors associated with such discrepancies compared with concordant HL.

Results Among 14345 participants, 1876 (13.1\%) had underestimated HI while 733 (5.1\%) had overestimated HI. Multivariable models revealed that participants who had discrepancies between SHD and AHL were less likely to have older age (OR: 0.979, $95 \% \mathrm{Cl}: 0.967$ to 0.991 for the underestimated HI, OR: $0.905,95 \% \mathrm{Cl}: 0.890$ to 0.921 for the overestimated $\mathrm{HI}$ ) and tinnitus (OR: $0.425,95 \% \mathrm{Cl}: 0.344$ to 0.525 for the underestimated $\mathrm{HI}$ and $\mathrm{OR} 0.523,95 \% \mathrm{Cl}$ : 0.391 to 0.699 for the overestimated $\mathrm{HI}$ ) compared with those who had concordant $\mathrm{HI}$. Exposure to occupational noise (OR: $0.566,95 \% \mathrm{Cl}: 0.423$ to 0.758 ) was associated with underestimated $\mathrm{HI}$, and medical history of hypertension (OR: $1.501,95 \% \mathrm{Cl}: 1.061$ to 2.123) and depression (OR: $1.771,95 \% \mathrm{Cl}: 1.041$ to 3.016) was associated with overestimated $\mathrm{HI}$. Conclusion Age, tinnitus, occupational noise exposure, hypertension and depression should be incorporated into evaluation of hearing loss in clinical practice.

\section{Strengths and limitations of this study}

- This study was based on a nationwide large-scale cross-sectional survey.

- We analysed only participants who had normal tympanic membranes to exclude participants who have undergone a previous hearing evaluation.

- We used definition of hearing loss as mean hearing threshold of $>25 \mathrm{~dB}$ HL measured at 0.5, 1, 2 and $4 \mathrm{kHz}$ in the better ear in accordance with the WHO definition (World Health Organization 2014).

- Multivariable logistic analysis was performed using both auditory and non-auditory factors including personal, socioeconomic, psychological and health-related factors.

- Because the survey did not assess the history of hearing evaluation for each participant, this might have influenced discrepancy between self-reported hearing and audiometry.

\section{INTRODUCTION}

Hearing is usually assessed in the clinic by using pure-tone audiometry to measure the smallest detectable level of pure tone at several frequencies, typically in the range of $0.5-8 \mathrm{kHz}$. Sometimes, the use of self-reported hearing measurements is attractive in occupational health screening programmes or a large-scale epidemiologic survey due to the costs and time constraints of audiometric measurements. However, discrepancies between self-reported hearing and pure-tone thresholds have been reported in multiple studies. ${ }^{1-11}$ Therefore, it is necessary to understand prevalence of this discrepancy and various factors affecting the accuracy of self-reported hearing when using as a surrogate measurement of audiometry.

Previous studies have reported that accuracy of self-reported hearing difficulty (SHD) is associated with auditory factors (eg, 
degree of hearing loss, frequencies of hearing loss and middle ear infection) $)^{5-79101213}$ as well as demographic factors. ${ }^{3} 571415$ However, these studies have mainly focused on elderly populations ${ }^{381114}$ or SHD with normal audiogram. ${ }^{17}$ Few studies have focused on the non-auditory factors (socioeconomic factors, psychological factors, healthcare utilisation or other personal information) that might influence the self-reported hearing assessment in a large population of various ages. Although a study has recently reported discrepancy between self-reported hearing and audiometry, ${ }^{5}$ this study included participants with abnormal tympanic membrane (TM) findings such as perforation, cholesteatoma or effusion. Because individuals who have abnormal TM are more likely to have undergone a previous hearing evaluation, this might have influenced self-reported hearing and also discrepancy from audiometry.

The primary aim of this study was to evaluate the prevalence of discrepancy between SHD and audiometrically measured hearing loss (AHL) in terms of overestimation or underestimation in a population with normal TMs based on national survey data. We also comprehensively investigated whether non-auditory metrics such as socioeconomic factors, psychological factors, medical history, healthcare utilisation and other personal information could affect the accuracy of SHD and types of discrepancy.

\section{METHODS}

\section{Data source}

This study used data from the fifth Korea National Health and Nutrition Examination Survey (KNHANES). The KNHANES is a nationwide cross-sectional survey conducted annually by the Korea Centers for Disease Control and Prevention (KCDC) to investigate health and nutritional status of a representative Korean population. ${ }^{16}$ Every year, about 10000 individuals in 3840 households are selected from a panel to represent the population through a multistage clustered and stratified random sampling method based on National Census Data. A total of 576 survey areas were drawn from the population and housing census by considering the proportion of each subgroup. The participation rate of selected households was about $80 \%$. The survey manuals and microdata of KNHANES are available in public through the official website of KNHANES (http://knhanes.cdc.go.kr).

\section{Study population}

From 2010 to 2012, a total of 23621 individuals (8313 in 2010, 7887 in 2011 and 7421 in 2012) agreed to participate in health surveys. Among participants $>19$ years of age, we included participants who completed hearing questionnaire, audiometric measurement and examination of TMs. As individuals with abnormal TM are more likely to have correct information on their hearing status from the prior hearing tests, we excluded participants with abnormal TM, and whose information on outcome variables was missing.

\section{Hearing questionnaire and audiometric measurement}

Participants were first asked about their perceived HD. In detail, participants were asked to rate their difficulty in hearing with a survey question: 'Which sentence best describes your hearing status (while not using hearing aids)?', and to choose an answer for the question: (1) 'Don't feel difficulty at all,' (2) 'A little bit difficult', (3) 'Very difficult' and (4) 'Can't hear at all'. SHD was indicated when the response was (2), (3) or (4).

Pure tone air-conduction threshold was measured in a double-walled sound booth (CD-600, Sontek, Paju, South Korea) using an audiometer (SA-203, Entomed AB, Malmö, Sweden). A TDH39P Phone type headphone $(10 \mathrm{Ohm})$ was used. Calibration of the audiometer was carried out annually according to the user's manual. The ambient noise level measured inside the booth under maximal noisy conditions in the survey unit met the ISO 8253-1 standard. Otolaryngologists who had been trained to operate the audiometer provided instructions to participants and obtained audiometric data. Air conduction thresholds were measured at $0.5,1,2,3,4$ and $6 \mathrm{kHz}$ in accordance with the American National Standards Institute standard. ${ }^{17}$

Hearing loss (HL) in this study was defined as the mean air conduction hearing thresholds $>25 \mathrm{~dB}$ HL at $0.5,1,2$ and $4 \mathrm{kHz}$ in the better ear. Discrepancy between self-reported hearing and audiometry was classified in terms of underestimated and overestimated hearing impairment (HI). Underestimation of HI was defined as having AHL without SHD. Likewise, overestimation of $\mathrm{HI}$ was defined as having SHD without AHL. Concordant HI was defined as having both AHL and SHD.

\section{Otologic examination and questionnaires}

An ear examination was conducted with a $4 \mathrm{~mm} 0^{\circ}$-angled rigid endoscope attached to a Charge-Coupled Device camera by trained otolaryngologists. Endoscopic examination was performed to identify abnormal TM findings such as perforation, cholesteatoma (including retraction pocket) and otitis media with effusion (including the presence of a ventilation tube). Trained otolaryngologists categorised both TMs into the following three groups: normal, abnormal and could not examine. Only participants with normal TMs on both sides were included in this study.

Participants were asked about their tinnitus experiences using the following question: 'During the past year, did you ever hear a sound (buzzing, hissing, ringing, humming, roaring, machinery noise) originating in your ear?'. Examiners were instructed to record either 'yes' or 'no'. If a participant reported that they heard an odd or unusual noise at any time in past years, examiners recorded 'yes'. Participants were also asked about their experience with occupational noise exposure. They were instructed to record either 'yes' or 'no' for the question 
'Have you ever worked more than 3 months in the place where you have to speak loudly to communicate with others because of noisy sound?'

\section{Outcome variables}

Age, sex, smoking status, alcohol consumption, marital status, waist circumference $(\mathrm{cm})$ and body mass index $\left(\mathrm{kg} / \mathrm{m}^{2}\right)$ of each participant were collected and categorised as personal factors in this study. Smoking status was divided into three groups: never smoked, past smoker and current smoker. The participants were asked to self-report to question 'Do you smoke now?'. If the participant smoked in the past but did not smoke now, it was classified as a past smoker. Alcohol consumption was divided into two groups according to their drinking frequency during the last year: non-drinker and drinker. The question was 'How often do you drink alcohol in the last year?'. The participants who had never drunk at all during the last year were classified as non-drinker, while others were classified as drinker.

A non-drinker was defined as a participant who had never drunk during the last year. Marital status was divided into two groups through the questionnaire: ever married and never married. The marital status question was 'Have you been married?'. Ever married included participants married at the time of survey, separated, widowed or divorced.

To evaluate socioeconomic factors, monthly income, education level and employment status were assessed. Participants answered an open-ended question on income: 'What is your average monthly income including salaries, property income, pension, government subsidies and allowance?'. Monthly income indicates equalised monthly household income and was calculated by dividing total family income by the square root of the number of household members. Monthly income was classified into quartiles to determine monthly income level: lower, lower middle, upper middle and upper. With regard to educational level, the participants were asked the level at which their education was completed, which was classified into four educational categories: completion of elementary school, middle school, high school and post-secondary school. Education level was re-divided into two groups: less than high school and high school or more. Employment status was divided into employed and unemployed groups. The participants answered either 'yes' or 'no' to the question 'Have you ever worked more than 1 hour for the last week for income, or worked as unpaid family worker for over 18 hours? (The temporary leave status is also included if you have worked.)'

Quality of life was measured using Euro Qol-5D (EQ-5D) consisting of a health-status descriptive system (EQ-5D) and a visual analogue scale (EQ-VAS). EQ-5D is a standard tool used to measure patient's health status in the following five dimensions: mobility, self-care, usual activities, pain/discomfort and anxiety/depression. ${ }^{18} 19$ Each dimension has three grades of severity: no problem (score of 1), moderate problem (score of 2) or serious problem (score of 3). EQ-5D index is calculated from EQ-5D score by applying a formula that assigns weights to each grade in each dimension. This formula differs among nations because it is based on the value of EQ-5D of the population. ${ }^{20}$ KNHANES algorithm was used to calculate the EQ-5D index in the present study. The EQ-5D index ranged from 1 (best health) to 0 (equivalent to death) or -0.171 (worse than death). Next, participants described their own health status using a VAS ranging from 0 (worst imaginable health) to 100 (best imaginable health) presented as EQ-VAS.

To evaluate psychological factors, self-reported health status and body shape perception were assessed. Self-reported health status was categorised into three answers: good, fair and poor. The question was 'What do you usually think about your health?'. Participants were asked to report their body shape perception as 'too thin', 'just right' or 'too fat'. The question was 'What do you think of your body weight status?'. Self-reported stress and depression levels were also assessed. Participants were asked about their stress level using the following question 'How much do you feel stress in ordinary life?'. They were instructed to report one of the following responses to the question: 'extremely stressed', 'quite stressed', 'a little bit stressed' and 'not stressed at all'. The responses were re-categorised into 'low level (not stressed at all or a little bit stressed)' or 'high level (extremely or quite stressed)'. To assess the self-perceived level of depression, participants answered either 'yes' or 'no' to the question 'Have you felt sorrow or despair that has affected your daily life for more than 2 weeks continuously during the past year?'

To evaluate health-related factors, physical activity, the use of medical service and current disease were assessed. The intensity of the physical activity was categorised as vigorous, moderate and light. Examples of vigorous intensity physical activities were soccer, basketball, aerobics, running, fast cycling and fast swimming. Moderate physical activities included cycling at a regular pace, swimming at a regular pace, slow swimming, noncompetitive volley ball and doubles tennis. Walking slowly or at a moderate pace for the use of public transportation were included in the light physical activity. We used the guidelines suggested by Noh $e t a l^{21}$ to divide the participants into exercising and non-exercising groups based on the number of days and hours in which they took part in physical activity. The intensity of the physical activity was based on the physical activity recommendations of the Centers for Disease Control and Prevention and the American College of Sports Medicine. These activities were categorised as follows: those who perform vigorous-intensity activity for a minimum of $20 \mathrm{~min}$ at least 3 days each week; those who perform moderate-intensity physical activity for a minimum of $30 \mathrm{~min}$ at least 5 days each week and those who perform light-intensity activity for a minimum of $30 \mathrm{~min}$ for at least 5 days weekly. Individuals who did not exercise regularly were placed into the non-exercising group. Medical services evaluated restriction of medical service, health screening and medical history. 
The participants were asked to answer either 'yes' or 'no' about the restricted use of medical service. The question was 'Have you ever been unable to go to the clinic (except for dentistry) during the past year?'. To assess the health screening status, the participants answered either 'yes' or 'no' to the question 'Have you ever had a health checkup for health during the last two years?' Participants were also asked about their current disease diagnosed by a medical doctor. They answered either 'yes' or 'no' to questions about current disease. Among the various disease lists, histories of hearing-related diseases such as obesity, hypertension, myocardial infarction, angina, asthma, depression, renal failure and diabetes mellitus were selected as variables. ${ }^{22} 23$

According to the standard protocol, systolic blood pressure (BP) and diastolic BP were measured by trained nurses using a mercury sphygmomanometer (Baumanometer Desk model; Baum, Amherst, New York, USA) on the right arm of the subject while sitting after taking at least $5 \mathrm{~min}$ of rest. BP was measured three times and the second and third measurements were averaged. Blood and urine samples were collected in the morning after fasting for at least 8 hours. Fasting blood samples and spot urine samples were processed, refrigerated immediately, and transported in cold storage to a central laboratory (Neodin Medical Institute, Seoul, Korea). All samples were analysed within 24 hours after transportation. Total cholesterol, high-density lipoprotein (HDL) cholesterol, triglyceride, haemoglobin, haematocrit, blood urea nitrogen and serum creatinine levels were measured with a Hitachi Automatic Analyzer 7600 (Hitachi, Tokyo, Japan). Urine protein and glucose levels were measured using a dipstick in a spot urine sample.

\section{Statistical analysis}

All statistical analyses were performed by taking account of weights from a complex sampling design according to the guideline for analysis of KNHANES data. The KCDC has published guideline for analysis through the official website of KNHANES (http://knhanes.cdc.go.kr). The survey design created a sample weight assigned to each sample individual through the following three steps so that the total sample would represent the population (on average) for 2010-2012 period: calculating the base weight of the inverse of the final probability an individual being selected, adjusting for non-response and post-stratification adjustment to match previous census population control totals. Weights in 2010, 2011, 2012 surveys were combined, and the average weight (sum of weight for each year/3) was calculated. Statistical analyses were performed using SAS V.9.4 (SAS Institute).

Logistic regression or linear regression was used to evaluate factors associated with discrepancies between SHL and AHL. Variables found to have possible association in univariable analysis $(\mathrm{p}<0.20)$ were entered into the multivariable analysis model. Serologicaldata were not entered into the multivariable analysis model due to a significant number of missing data. In this study, the population group was classified into three categories: participants who had overestimated HI, underestimated HI and concordant HI. To evaluate factors associated with underestimated HI, we compared participants with underestimated HI and concordant HI. We also compared participants with overestimated $\mathrm{HI}$ and concordant HI to evaluate factors associated with overestimated HI. The $p$ values were obtained two-sided. Bonferroni's correction was applied to the $p$ value and the corresponding CI due to multiple testing. Statistical significance was considered when adjusted $\mathrm{p}$ value was less than 0.05 .

\section{Patient and public involvement}

Participants and the public were neither involved in designing the study or developing the research questions, nor were they involved in analysing or interpreting the findings. There are no plans for the study results to be disseminated directly to participants.

\section{RESULTS}

\section{Basic characteristics of study population}

A total of 25094 Korean citizens participated in the KNHANES from 2010 to 2012. Of them, 16727 participants aged $\geq 19$ years completed the hearing questionnaire and audiometric measurement. After excluding participants with abnormal TM and missing data, a total of 14345 participants were ultimately eligible for this study. The mean \pm SDage of the study population was $49.2 \pm 16.1$ years (ranged from 19 to 97 ). The study population consisted of $42.5 \%$ males and $57.5 \%$ females.

\section{Prevalence of discrepancies between self-reported hearing and audiometry}

Of the 14345 participants with normal TMs, 3001 (20.9\%) participants had AHL and 1858 (13.0\%) had SHD. Table 1 shows the percentage and prevalence of discrepancies between self-reported hearing and audiometry. Of the 3001 participants with AHL, $62.5 \%(n=1876)$ reported no SHD. On the other hand, 733 (39.5\%) of 1858 participants with SHD had no AHL (mean audiometric thresholds $\leq 25 \mathrm{~dB}$ HL in the better ear). That is, the prevalence of underestimated and overestimated HI was $62.5 \%$ and $39.5 \%$, respectively. The prevalence of discrepancies between self-reported hearing and audiometry was $18.2 \%(n=2.609)$.

\section{Factors associated with underestimated hearing impairment}

A total of 3001 participants who had bilateral HL (mean hearing thresholds $>25 \mathrm{~dB} \mathrm{HL}$ at $0.5,1,2$ and $4 \mathrm{kHz}$ ) were analysed to evaluate factors associated with underestimated HI using linear and logistic regression analyses. Results are shown in table 2. In univariable analyses, age, alcohol consumption, education, employment status, quality of life, self-reported health status, depressive mood, restricted use of medical service, hospital visit, history of myocardial infarction, angina, asthma, tinnitus, occupational noise exposure, diastolic BP and 
Table 1 Percentage and prevalence rates of discrepancy between self-reported hearing and audiometry

\begin{tabular}{|c|c|c|c|}
\hline Audiometry & Hearing difficulty & No difficulty & Total \\
\hline Hearing loss & $1125(\mathrm{~A})$ & $1876(B)$ & $3001(A+B)$ \\
\hline Total & $1858(A+C)$ & $12487(\mathrm{~B}+\mathrm{D})$ & $14345(A+B+C+D)$ \\
\hline
\end{tabular}

Percentage of discrepancy $(\%)=18.2 \%[(B+C) /(A+B+C+D)]$.

Underestimation of hearing impairment $=62.5 \%[B /(A+B)]$.

Overestimation of hearing impairment $=39.5 \%[C /(A+C)]$.

blood urea nitrogen were significantly associated with underestimated HI. In multivariable analysis, participants who underestimated HI showed significantly decreased age (OR: $0.979,95 \%$ CI: 0.967 to 0.991 ) compared with those who had both AHL and SHD. Also, participants who underestimated HI were less likely to have tinnitus (OR: $0.425,95 \% \mathrm{CI}: 0.344$ to 0.525 ) or exposure to occupational noise (OR: $0.566,95 \%$ CI: 0.423 to 0.758 ) compared with those who showed concordant HI.

\section{Associated factors with overestimated hearing impairment}

A total of 1858 participants who had SHD were analysed to investigate factors associated with overestimated HI. Results of univariable and multivariable analyses are shown in table 3. In univariable analysis, age, sex, smoking, alcohol consumption, waist circumference, monthly income, marital status, education level and employment status were significantly associated with overestimated HI compared with those who had both SHD and AHL. For quality of life factors, EQ-5D subscales such as physical activity about mobility, self-care, and usual activity, EQ-5D index and EQ-VAS were significantly associated with overestimated HI. For psychologic factors, self-reported health status, body shape perception and amount of stress in life were significantly associated with overestimation of HI. Overestimation of HI was also significantly associated with vigorous and moderate physical activity, hospital visit and history of hypertension, angina, depression, diabetes mellitus and tinnitus. Systolic BP, HDL cholesterol, blood urea nitrogen and serum creatinine levels were also significantly associated with overestimated HI. In multivariable analysis, participants who overestimated HI showed significantly decreased age (OR: $0.905,95 \%$ CI: 0.890 to 0.921 ) compared with those who had concordant HI. Participants who overestimated HI were more likely to have hypertension (OR: 1.501, 95\% CI: 1.061 to 2.123) and depression (OR: 1.772, 95\% CI: 1.041 to 3.016) but less likely to report tinnitus (OR $0.523,95 \%$ CI: 0.391 to 0.699) compared with those who had both SHD and AHL.

\section{DISCUSSION}

This cross-sectional survey of Korean population aged $\geq 19$ years found that $18.2 \%$ of participants had a discrepancy between their SHD and AHL. Most (71.9\%) of these participants had AHL but no SHD (underestimated
HI) while the rest $(28.1 \%$ ) had SHD but no AHL (overestimated HI, table 1). The accuracy of hearing assessments in the present study $(81.8 \%)$ was higher than that reported in elderly population of USA $(71.8 \%),{ }^{3}$ but similar to that reported in the general population of Australia (82\%). ${ }^{6}$ Previously, Kim et al categorised the self-reported hearing into three categories (no difficulty, a little difficulty and much difficulty) and classified the mean pure-tone threshold of the better ear into three groups $(<25 \mathrm{~dB}, \geq 25 \mathrm{~dB}$ and $<40 \mathrm{~dB}$, and $\geq 40 \mathrm{~dB})$. When the participants of previous study ${ }^{5}$ were reclassified as in our study, the accuracy of hearing assessments was slightly higher $(83.2 \%)$ than our result. In addition, our result showed that $5.1 \%$ (733 of 14325 ) of participants reported overestimated HI and $13.1 \%$ (1876 of 14325 ) reported underestimated HI. However, reclassified results in Kim et al showed that $6.3 \%$ (1237 of 19 642) of participants reported overestimated $\mathrm{HI}$ and $10.5 \%$ (2059 of 19 642) of participants reported underestimated HI. Although present study and Kim et al analysed using same dataset, participants with abnormal TMs were excluded in our study, but included in Kim et al. Thus, differences in prevalence can be explained by the fact that individuals who have abnormal TM are more likely to report SHD and are more likely to have undergone a previous hearing evaluation.

Our results showed that both non-auditory factors (demographic factors and medical histories) and auditory factors (tinnitus and occupational noise exposure) were associated with discrepancy between self-reported hearing and audiometry in multivariable analysis. For demographic factors, participants who underestimated or overestimated their HI were significantly younger compared with participants who had concordant HI (tables 2 and 3). It is well known that audiometric HL dramatically increases with increasing age ${ }^{23}$ SHD is also increased with age as difficulty of speech understanding in adverse listening conditions increases ${ }^{24}$ due to decreased synaptic loss, ${ }^{25}$ working memory capacity ${ }^{27}$ or impaired temporal processing. ${ }^{128}$ Our reference group was defined as participants who had both SHD and AHL (concordant $\mathrm{HI}$ ), so it is highly likely that older participants will have both SHD and AHL. Therefore, it is not surprising that younger participants were less likely to have SHD among participants with audiometric HL (table 2) and had fewer 


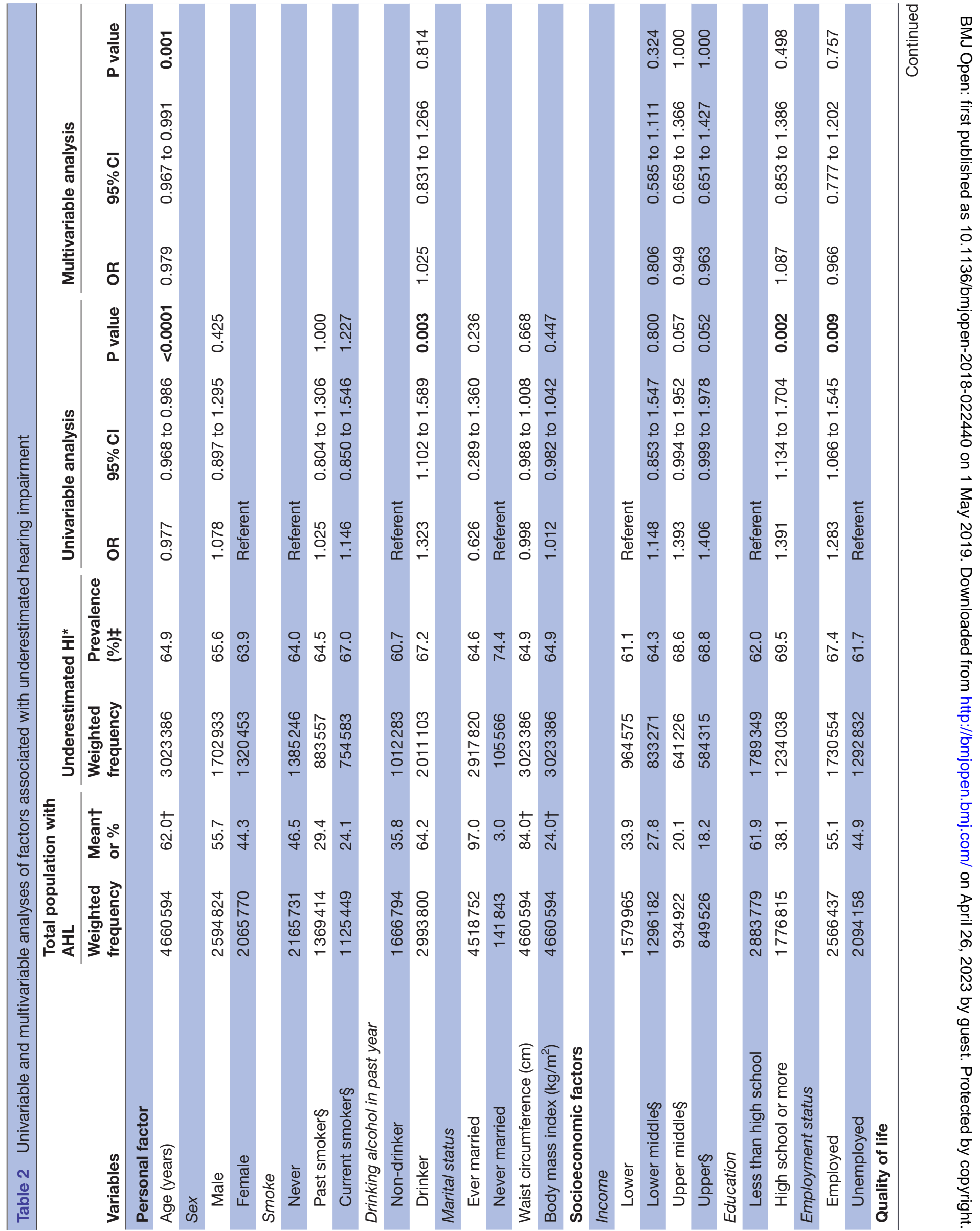




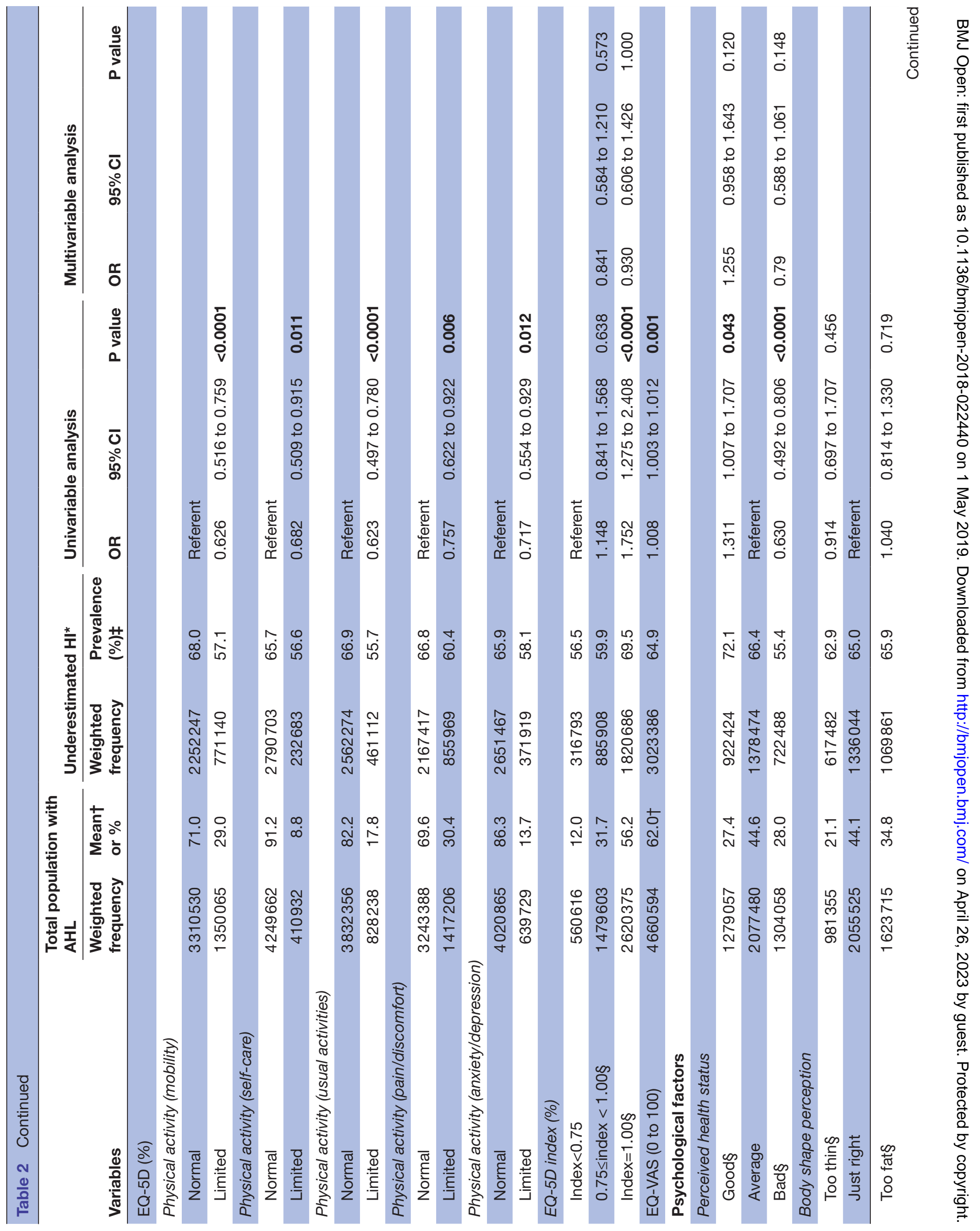




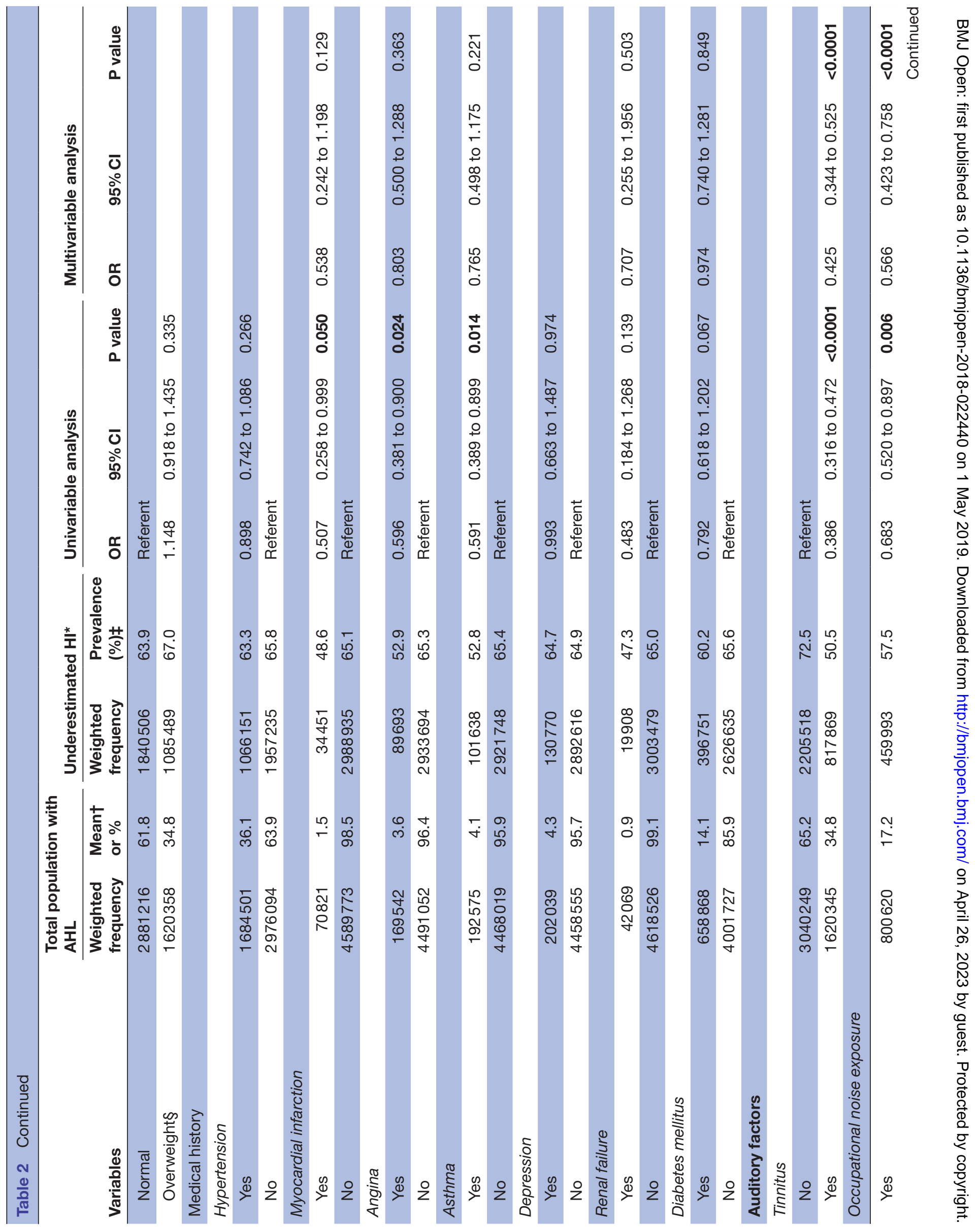




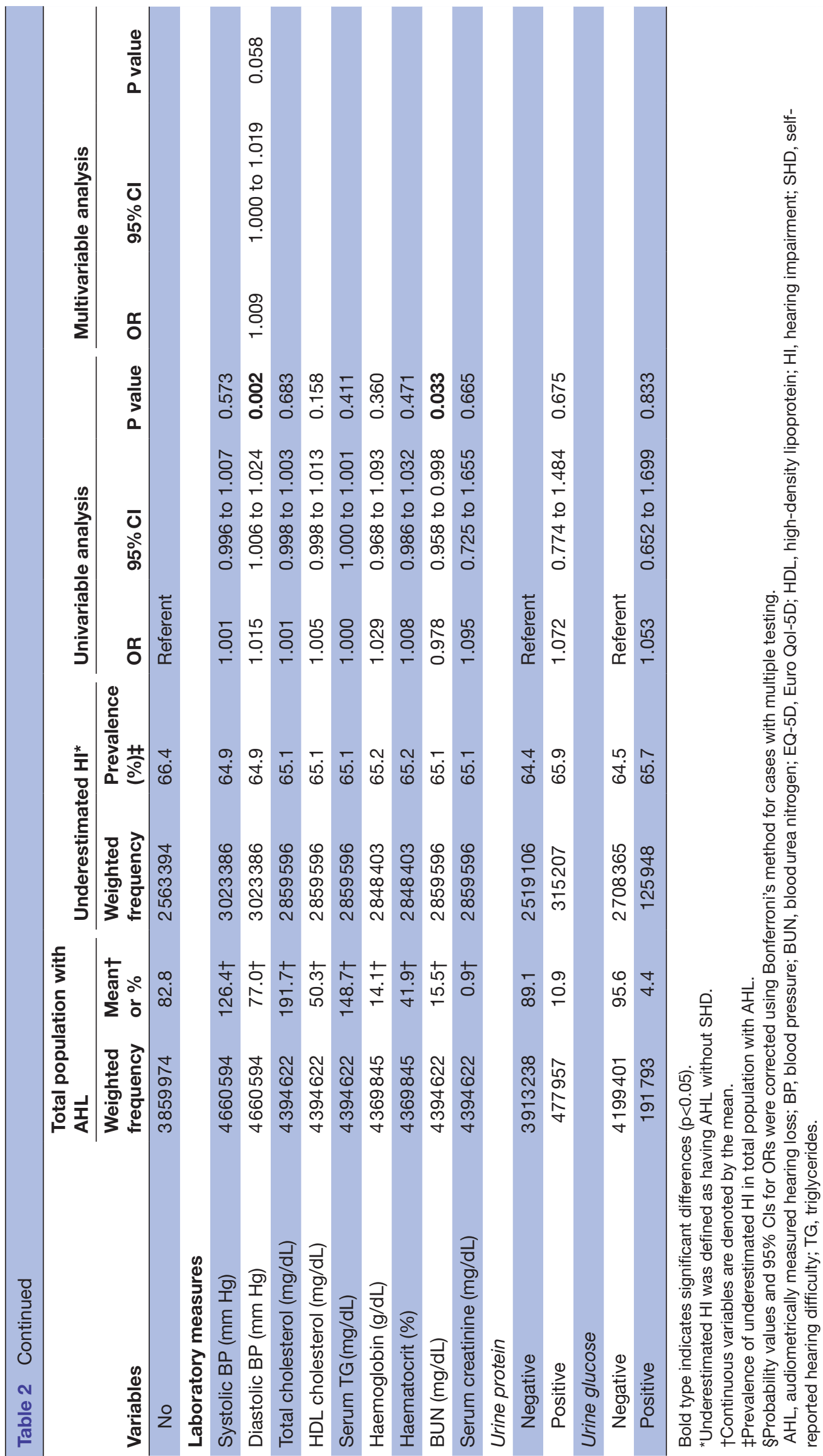




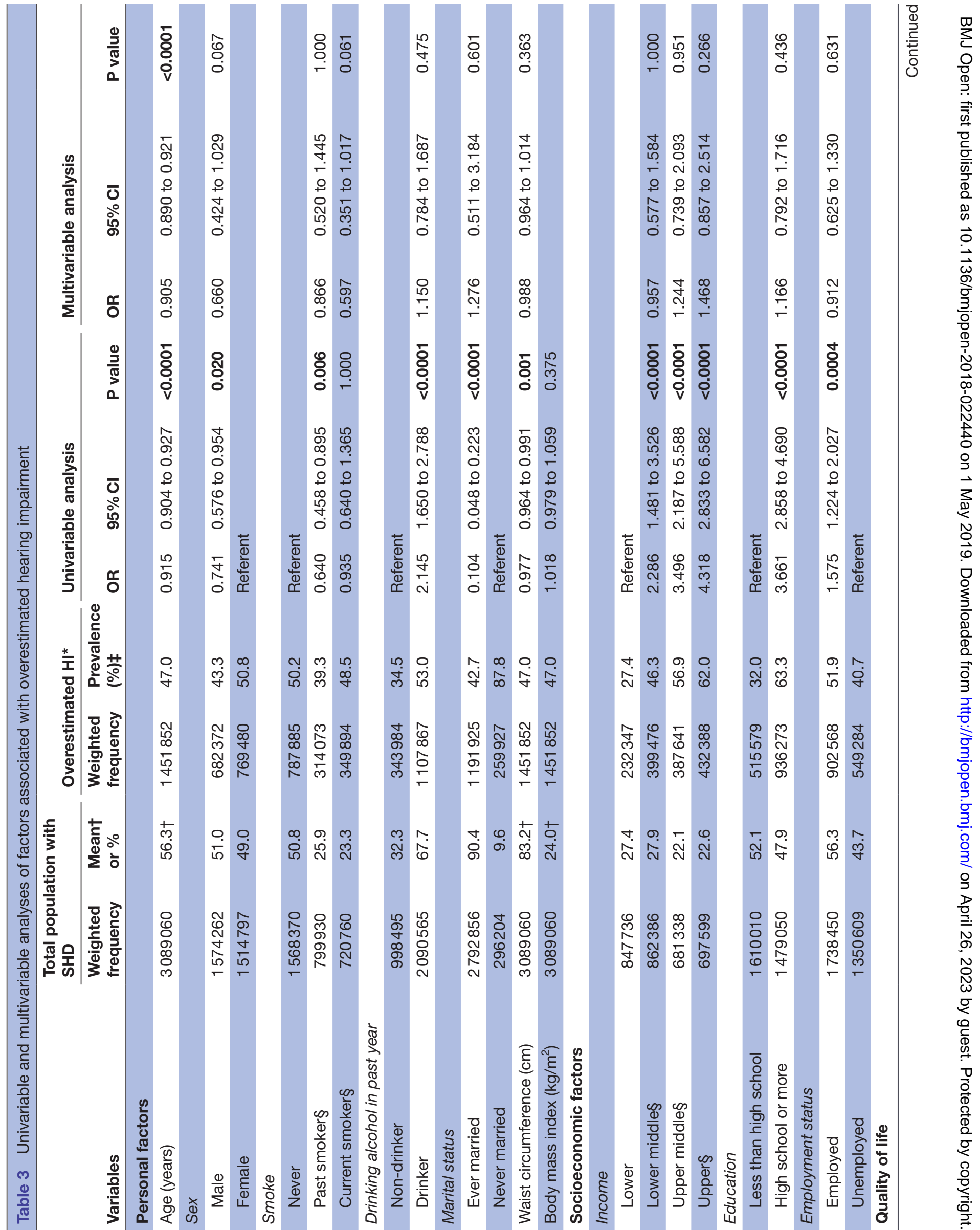




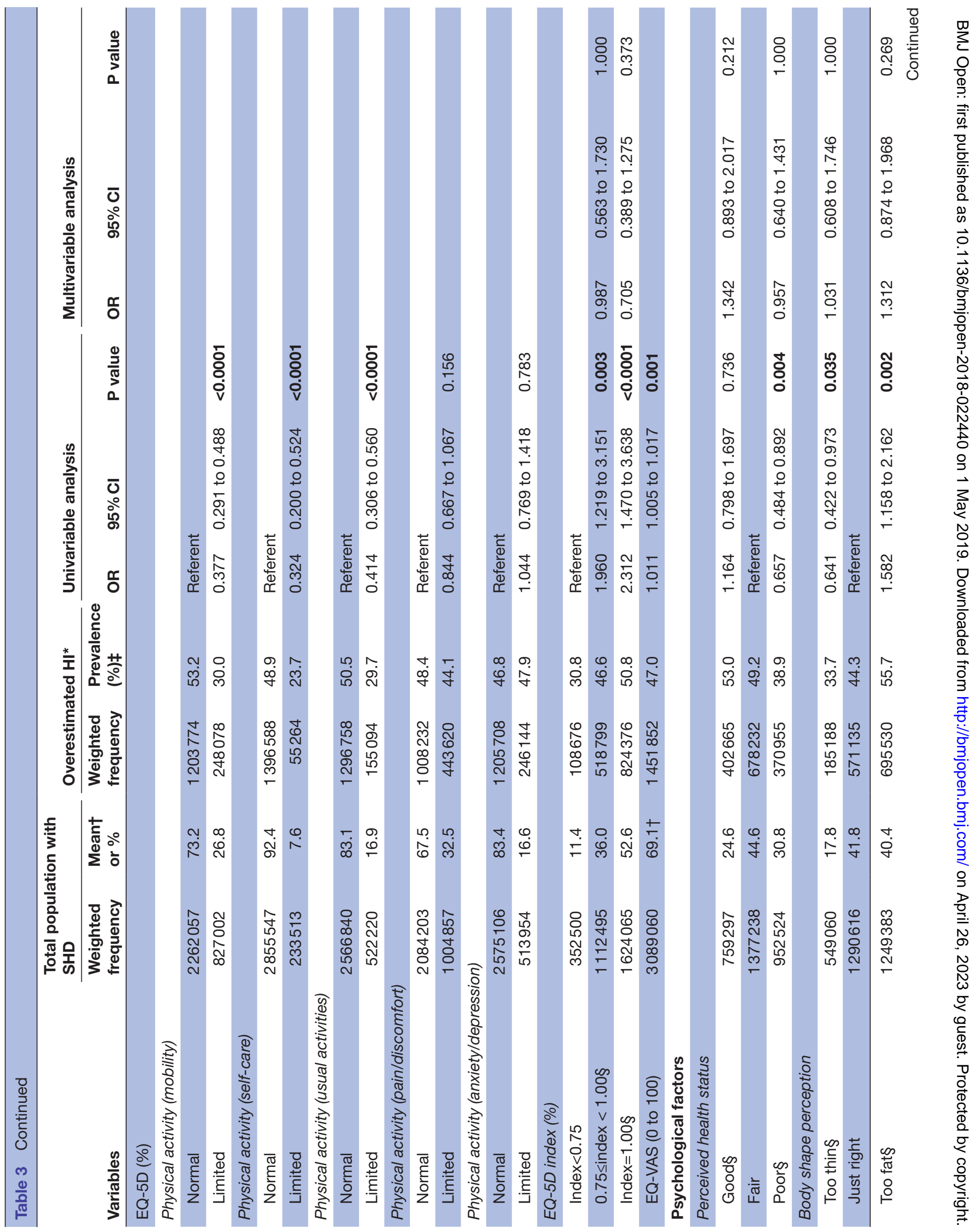




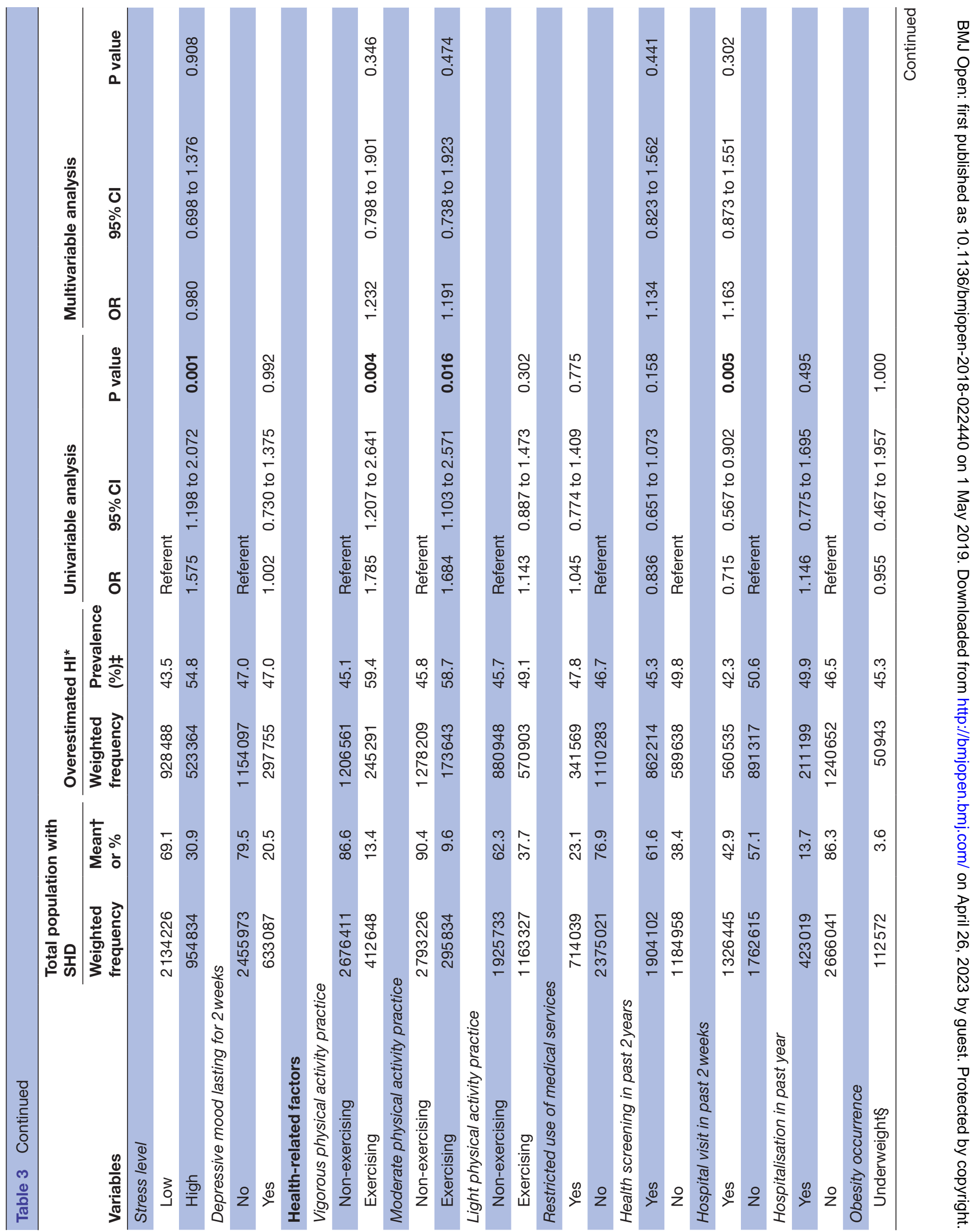




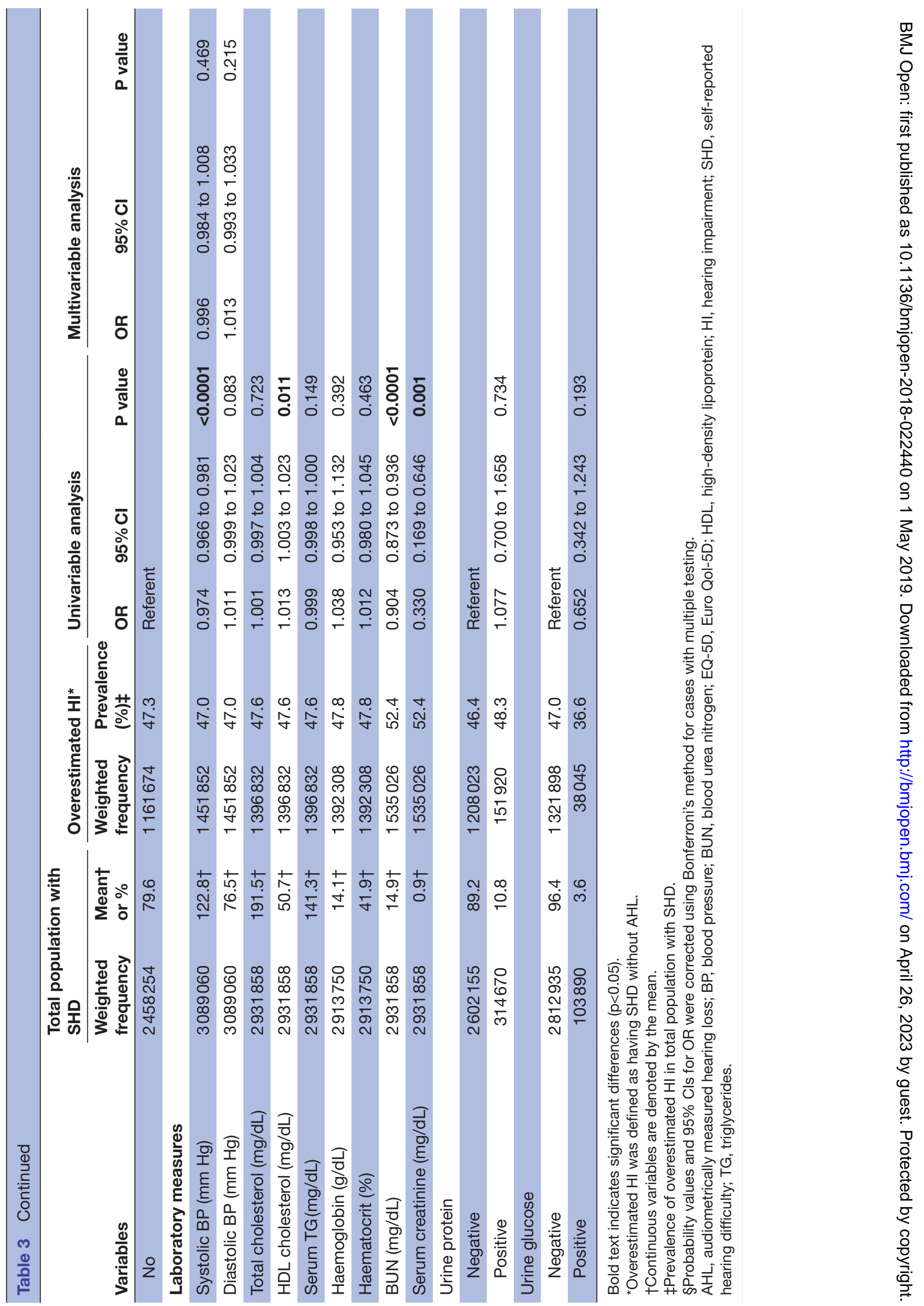


audiometric HL among participants with SHD (table 3). In contrast to our result, Kamil $e t a \vec{l}^{3}$ has reported that old age was related to underestimation of HI. The contradictory result between our study and Kamil et al may be due to the fact that younger people who underestimated HI were not included because they examined participants aged $\geq 50$ years. Among 2609 participants with discrepancy between SHD and AHL in this study, underestimated HI was more prevalent in older participants than overestimated HI, and it might be attributed to a tendency of older population to consider their HL to be 'normal' for their age. ${ }^{3}$

For medical-related factors, participants who overestimated their HI significantly had more hypertension and depression than those who had concordant HI (table 3). Because hypertension is known to increase the risk of cochlea damage possibly through malfunction of the stria vascularis, ${ }^{29}$ it might be related to early development of preclinical HL in auditory way. Also, hypertension and depression may influence the SHD in non-auditory way. Subjects with hypertension have worse overall health than subjects without hypertension, which in turn has been shown to be associated with an increased likelihood of reporting HD. ${ }^{30}$ Studies have suggested that personality traits of neuroticism had a more adverse perception of their HD, ${ }^{3132}$ and it is widely known as an important factor that influences depression. ${ }^{33}$ Accordingly, hypertension and depression may lead to an increased perception of HD. Moreover, as the present study is cross-sectional, it cannot be excluded that hypertension and depression is a result of SHD.

For auditory factors, tinnitus and occupational noise exposure were associated with concordant HI (tables 2 and 3). It is possible that these participants had an audiometric assessment for their tinnitus or occupational health screening programme and had known about their hearing status. Participants who had been exposed to occupational noise tended to have less underestimated HI regardless of tinnitus (table 2). As they are more likely to have severe HL than other participants, the severity of HL may affect SHD. ${ }^{9}$

Although a similar study from same dataset has been recently reported, ${ }^{5}$ our study has several significant differences in approach. First, we excluded data from participants with abnormal TM who are more likely to have undergone a previous hearing evaluation. Second, we excluded normal hearing population with normal audiometry $(<25 \mathrm{~dB})$ and without SHD in the reference group, and confined the concordant HI group to those who showed both SHD and AHL as reference. However, Kim et $a \tilde{l}^{\tilde{b}}$ had the concordance group including normal hearing population as reference. Because a large number of normal hearing people (93\%) were included in their reference group, their analysis is likely to be biased by factors related to SHD or AHL, rather than focusing on the discrepancy between subjective hearing assessment and audiometry itself. Subgroup analysis for participants with $\geq 25 \mathrm{~dB}$ in Kim et a $\tilde{l}$ showed that age, sex, education, occupation and stress were not associated with the discrepancy between subjective hearing assessment and audiometric thresholds. Lastly, this study analysed more variables including smoking status, alcohol consumption, waist circumference, body mass index, monthly income, marital status, quality of life, self-reported health status, body shape perception, noise exposure, physical activity, the use of medical service, current disease and serological data. Therefore, we expected that this study could provide more comprehensive information related to discrepancy between SHD and AHL.

In summary, the prevalence of discrepancy between SHD and AHL was $18.2 \%$ in South Korea. Age, medical histories of hypertension and depression, tinnitus and occupational noise exposure were associated with inconsistent results between self-reported and audiometrically measured hearing assessment in multivariable analysis. Understanding the factors related to self-reported hearing will assist clinicians in interpreting subjective reports of hearing and using these data as a surrogate measure of audiometry. These factors need to be considered when determining whether to conduct a hearing test, even if the patients do not report an HI.

\section{Author affiliations}

${ }^{1}$ Department of Otorhinolaryngology-Head and Neck Surgery, Dankook University Hospital, Cheonan, Republic of Korea

${ }^{2}$ Department of Otorhinolaryngology-Head and Neck Surgery, Samsung Medical Center, Seoul, Republic of Korea

${ }^{3}$ Biostatistics and Clinical Epidemiology Center, Research Institute for Future Medicine, Samsung Medical Center, Seoul, Republic of Korea

Acknowledgements We thank the 150 residents of Otorhinolaryngology Departments of 47 training hospitals in South Korea and members of the Division of Chronic Disease Surveillance in Korea Centers for Disease Control and Prevention for collecting data in this survey and their dedicated work.

Contributors JEC and IJM designed research and wrote the main paper. S-YB and SWK collected and analysed data. Y-SC provided critical revision and discussed the results and implications and commented on the manuscript at all stages.

Funding The authors have not declared a specific grant for this research from any funding agency in the public, commercial or not-for-profit sectors.

Competing interests None declared.

Patient consent for publication Obtained.

Ethics approval All participants provided written informed consent before completing the survey. KNHANES followed the tenets of the Declaration of Helsinki for biomedical research. It was approved by the Institutional Review Board of the Korean Centers for Disease Control and Prevention (IRB No. 2010-02CON-21-C, 2011-02CON-06-C and 2012-01EXP-01-2C). Approval for this research study was obtained from the Institutional Review Board of Samsung Medical Center (IRB No. 2016-06-142).

Provenance and peer review Not commissioned; externally peer reviewed.

Data sharing statement Data are available from the Korea National Health and Nutrition Examination Survey (KNHANES) Data Access for researchers. Because annually, Korea Center for Disease Control and Prevention published the reports and microdata of KNHANES with survey manuals through the official website of KNHANES (http://knhanes.cdc.go.kr), all KNHANES data are de-identified and available to the public.

Open access This is an open access article distributed in accordance with the Creative Commons Attribution Non Commercial (CC BY-NC 4.0) license, which permits others to distribute, remix, adapt, build upon this work non-commercially, and license their derivative works on different terms, provided the original work is properly cited, appropriate credit is given, 
any changes made indicated, and the use is non-commercial. See: http:// creativecommons.org/licenses/by-nc/4.0/.

\section{REFERENCES}

1. Kiely KM, Gopinath B, Mitchell P, et al. Evaluating a dichotomized measure of self-reported hearing loss against gold standard audiometry: prevalence estimates and age bias in a pooled national data set. J Aging Health 2012;24:439-58.

2. Nondahl DM, Cruickshanks KJ, Wiley TL, et al. Accuracy of selfreported hearing loss. Audiology 1998;37:295-301.

3. Kamil RJ, Genther DJ, Lin FR. Factors associated with the accuracy of subjective assessments of hearing impairment. Ear Hear 2015;36:164-7.

4. Diao M, Sun J, Jiang T, et al. Comparison between self-reported hearing and measured hearing thresholds of the elderly in China. Ear Hear 2014;35:e228-32.

5. Kim SY, Kim HJ, Kim MS, et al. Discrepancy between self-assessed hearing status and measured audiometric evaluation. PLoS One 2017;12:e0182718.

6. Kirk KM, McGuire A, Nasveld PE, et al. Comparison of self-reported and audiometrically-measured hearing loss in the Australian Defence Force. Int J Audiol 2012;51:294-8.

7. Tremblay KL, Pinto A, Fischer ME, et al. Self-Reported Hearing Difficulties Among Adults With Normal Audiograms: The Beaver Dam Offspring Study. Ear Hear 2015;36:e290-9.

8. Sindhusake D, Mitchell P, Smith W, et al. Validation of self-reported hearing loss. The Blue Mountains Hearing Study. Int J Epidemiol 2001;30:1371-8.

9. Wiley TL, Cruickshanks KJ, Nondahl DM, et al. Self-reported hearing handicap and audiometric measures in older adults. J Am Acad Audiol 2000;11:67-75.

10. Spankovich C, Gonzalez VB, Su D, et al. Self reported hearing difficulty, tinnitus, and normal audiometric thresholds, the National Health and Nutrition Examination Survey 1999-2002. Hear Res 2017.

11. Hannula S, Bloigu R, Majamaa K, et al. Self-reported hearing problems among older adults: prevalence and comparison to measured hearing impairment. J Am Acad Audiol 2011;22:550-9.

12. Clinard CG, Tremblay KL, Krishnan AR. Aging alters the perception and physiological representation of frequency: evidence from human frequency-following response recordings. Hear Res 2010;264:48-55.

13. Swanepoel deW, Eikelboom RH, Hunter ML, et al. Self-reported hearing loss in baby boomers from the Busselton Healthy Ageing Study: audiometric correspondence and predictive value. J Am Acad Audiol 2013;24:514-21. quiz 29.

14. Chen DS, Genther DJ, Betz J, et al. Association between hearing impairment and self-reported difficulty in physical functioning. $J \mathrm{Am}$ Geriatr Soc 2014;62:850-6.

15. Ramkissoon I, Cole M. Self-reported hearing difficulty versus audiometric screening in younger and older smokers and nonsmokers. J Clin Med Res 2011;3:183-90.

16. Kweon S, Kim Y, Jang MJ, et al. Data resource profile: the Korea National Health and Nutrition Examination Survey (KNHANES). Int J Epidemiol 2014;43:69-77.
17. Institute. ANS. Specification for audiometers. ANSI S3.6-1996. New York: American National Standards Institute, 1996.

18. Sullivan PW, Ghushchyan V. Preference-Based EQ-5D index scores for chronic conditions in the United States. Med Decis Making 2006;26:410-20.

19. EuroQol Group. EuroQol - a new facility for the measurement of health-related quality of life. Health Policy 1990;16:199-208.

20. Choo J, Jeon S, Lee J. Gender differences in health-related quality of life associated with abdominal obesity in a Korean population. BMJ Open 2014;4:e003954.

21. Noh JW, Lee SA, Choi HJ, et al. Relationship between the intensity of physical activity and depressive symptoms among Korean adults: analysis of Korea Health Panel data. J Phys Ther Sci 2015;27:1233-7.

22. Seo YJ, Ko SB, Ha TH, et al. Association of hearing impairment with chronic kidney disease: a cross-sectional study of the Korean general population. BMC Nephrol 2015;16:154.

23. Hong JW, Jeon JH, Ku CR, et al. The prevalence and factors associated with hearing impairment in the Korean adults: the 2010-2012 Korea National Health and Nutrition Examination Survey (observational study). Medicine 2015;94:e611.

24. Humes LE. Understanding the speech-understanding problems of older adults. Am J Audiol 2013;22:303-5.

25. Barbee CM, James JA, Park JH, et al. Effectiveness of Auditory Measures for Detecting Hidden Hearing Loss and/ or Cochlear Synaptopathy: A Systematic Review. Semin Hear 2018;39:172-209.

26. Meister H, Schreitmüller S, Ortmann M, et al. Effects of Hearing Loss and Cognitive Load on Speech Recognition with Competing Talkers. Front Psychol 2016;7:301.

27. Füllgrabe $\mathrm{C}$, Rosen S. Investigating the Role of Working Memory in Speech-in-noise Identification for Listeners with Normal Hearing. Adv Exp Med Biol 2016;894:29-36.

28. Clinard CG, Tremblay KL. Aging degrades the neural encoding of simple and complex sounds in the human brainstem. J Am Acad Audiol 2013;24:590-9. quiz 643-4.

29. Przewoźny T, Gójska-Grymajło A, Kwarciany M, et al. Hypertension and cochlear hearing loss. Blood Press 2015;24:199-205.

30. Chang HP, Ho CY, Chou P. The factors associated with a selfperceived hearing handicap in elderly people with hearing impairment--results from a community-based study. Ear Hear 2009;30:576-83.

31. Cox RM, Alexander GC, Gray GA. Personality, hearing problems, and amplification characteristics: contributions to self-report hearing aid outcomes. Ear Hear 2007;28:141-62.

32. Jang Y, Mortimer JA, Haley WE, et al. Nonauditory determinants of self-perceived hearing problems among older adults: the role of stressful life conditions, neuroticism, and social resources. J Gerontol A Biol Sci Med Sci 2002;57:M466-9.

33. Navrady LB, Ritchie SJ, Chan SWY, et al. Intelligence and neuroticism in relation to depression and psychological distress: Evidence from two large population cohorts. Eur Psychiatry 2017;43:58-65. 